\title{
Molecular dynamic simulations of the sorption of toluene in a dry humic acid model: A preliminary study
}

\author{
Yang-hsin Shih ${ }^{\mathrm{a}, *}$, Jyh-shing Lin ${ }^{\mathrm{b}}$, Shian-chee $\mathrm{Wu}^{\mathrm{c}}$, Lien-feng Lee ${ }^{\mathrm{b}}$ \\ a Department of Soil and Environmental Sciences and Center of Nanoscience and Nanotechnology, \\ National Chung Hsing University, 250 Kuo-Kuang Rd., Taichung 402, Taiwan ROC \\ ${ }^{\mathrm{b}}$ Department of Chemistry, Tamkang University, Tamsui, Taiwan ROC \\ ${ }^{\mathrm{c}}$ Graduate Institute of Environmental Engineering, National Taiwan University, No. 71, \\ Chou Shan Road, Taipei 106, Taiwan ROC
}

Received 28 June 2005; received in revised form 25 September 2005; accepted 30 September 2005

Available online 15 November 2005

\begin{abstract}
Dynamical molecular properties like diffusion and sorption activation energy are of importance for a reliable prediction of the fate and remediation efficiency of organic contaminants in the subsurface. In this study, molecular dynamics, a computational technique aiming to describe the movement of molecules in time, has been used to study the sorption of organic contaminants in soil organic matter. The simulation results of the sorption kinetics and thermodynamic properties of toluene in humic acid are in reasonable agreement with experimental data. We believe that this technique will become an alternative and powerful tool not only to help understand the sorption mechanism at the molecular level but also to facilitate solving the problems of contaminated soil clean-up.
\end{abstract}

(C) 2005 Elsevier B.V. All rights reserved.

Keywords: Molecular dynamics; Soil organic matter; Humic acid; Sorption kinetics; Diffusion

\section{Introduction}

The sorption behavior of volatile organic compounds (VOCs) in humic substances plays important roles in controlling pollutant fate and the remediation of contaminated sites. There were many studies on mechanisms of the sorption process [1]. Previous works [2-10] have focused on examining sorption kinetics of VOCs with different types of environmental sorbents. These prior researches focused on studying sorption and diffusion behaviors in complex organo-mineral aggregates or soil humic substances at large length scales. Various rate models have been used to simulate contaminant uptake and release in a macroscopic scale. As to the mechanism controlling sorption dynamics, it still remains unclear due to the difficulty in observing its dynamic behavior at the microscopic level [11].

\footnotetext{
* Corresponding author. Tel.: +886 4 22854152; fax: +886 422862043 .

E-mail address: yhs@nchu.edu.tw (Y.-h. Shih).
}

Recently, molecular modeling techniques based on the development of fundamental physical theories and their applications to numerical simulation techniques are applied to environmental issues. For example, some researchers applied the molecular modeling techniques to explain the environmental phenomena, such as the persistency of toxaphene in mammals [12], the prediction of polychlorinated hydrocarbons from municipal waste incinerators [13], the sorption mechanisms of organic chemicals onto clays and minerals [14-18], the interactions of humic substances and minerals [19], and the interactions of contaminants and natural organic matter [20,21]. But to our knowledge there is very little molecular modeling work involved in sorption kinetics and thermodynamics study of a contaminant in humic substances.

Therefore, in this study we, will first use the molecular dynamics simulation technique to verify our method by comparing the kinetic results of simulation with the real experimental data estimated by our research group under dry conditions [5]. Then we will explore the possible mechanism governing the interaction between toluene, the target VOC, and humic acid. 
Finally, we will evaluate the sorption activation energy of toluene in humic acid.

\section{Computational methods and structural model}

\subsection{Structural model}

The building block structure used here for humic acid was described previously $[22,23]$ and was used to examine thoroughly the conformation by Sein et al. [24]. Sein et al. [24] indicated that multiple structural models of humic acid building blocks were in agreement with the experimental conditions of the studies. In this work, the basic building block structure of humic acid was produced using the $\mathrm{C}^{2}$ Builder modules [25]. The humic acid model consisting of seven monomers was built by adding one monomer after another gradually and at the same time the energy minimization calculations was carried out to assure its stable confirmation. Finally, the target compound, i.e. the toluene molecule, was inserted into the void space formed among chains within humic acid to establish our structural model as shown in Fig. 1. The unit-cell dimensions are $19.78 \AA \times 20.52 \AA \times 20.86 \AA$ and $\alpha=87.02^{\circ}, \beta=91.32^{\circ}$, and $\gamma=92.05^{\circ}$.

\subsection{Molecular dynamics simulation}

The motion and the applied force to atoms in molecular dynamic simulations are based on Newton's second law:

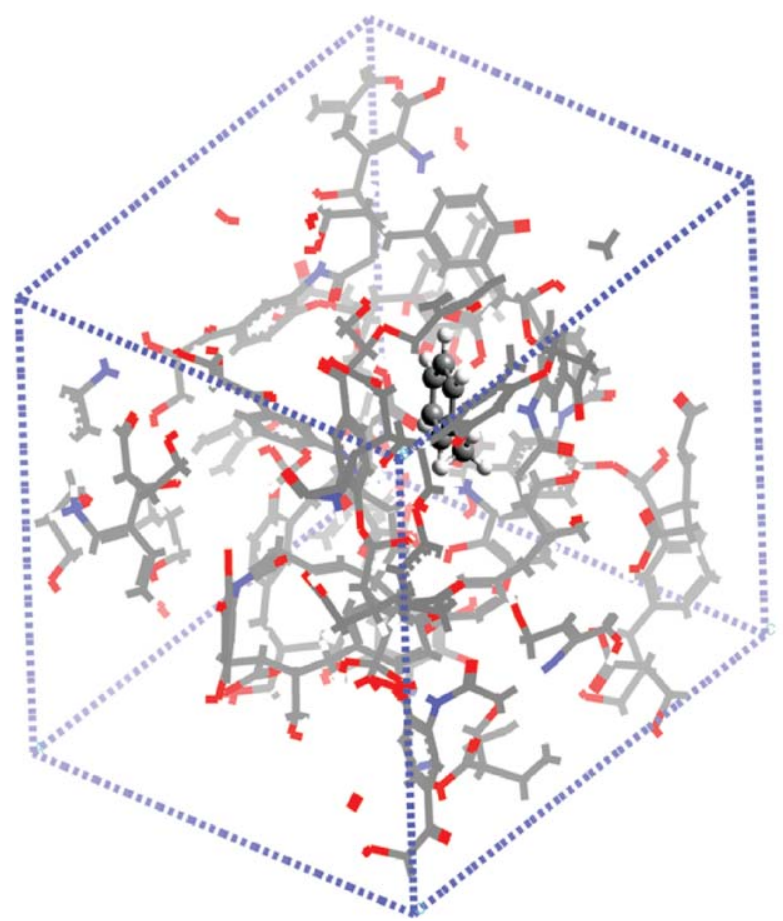

Fig. 1. The lowest-energy humic acid model containing a toluene molecule (the ball and stick form). Carbon atoms are gray, nitrogen atoms are blue, oxygen atoms are red, and hydrogen atoms are white. (For interpretation of the references to colour in this figure legend, the reader is referred to the web version of the article.)
$F=m a=m \frac{\mathrm{d}^{2} r}{\mathrm{~d} t^{2}}$

where $F$ is the force applied to the atom; $m$, the mass of the atom; $a$, the acceleration; and $r$, the position vector. The molecular dynamics model tracks the time evolution at the atomic level of ensemble of particles acting under specified interatomic forces by numerically solving the equation of motion in an iterative manner. Calculation of the forces of one specific system allows prediction of the positions and velocities of all atoms at the next time step based on Eq. (1). Subsequently, a new system configuration is obtained and the next iteration starts. The energy terms of one given system configuration and the velocities of the atoms can be calculated using intramolecular potential functions and intermolecular potential functions. The definitions of these energy terms and the values of the parameters constitute a force field. The force field descriptions obtained from the known structures are able to predict the energies of unknown structures. The interatomic force parameters used in this study were taken from the burchart 1.01-universal 1.02 force field distributed by MSI (Molecular Simulation Inc.) [25]. The burchart 1.01-universal 1.02 force field combines the burchart and universal force fields. In this field, the burchart force field treats the framework of the model and the universal force field treats the intramolecular and intermolecular interactions. The parameters for all non-bond interactions between the framework and the molecules in the model are derived from parameters from both force fields.

The simulation procedure used in this study was as follows. First, the simulations of the previous structural model of humic acid plus the toluene were run using the Dynamic module of the Cerius ${ }^{2}$ molecular modeling suite with the periodic boundary conditions. These simulations at 300,350 and $400 \mathrm{~K}$ were equilibrated for $50 \mathrm{ps}$ in the NVE ensemble and then run for $100 \mathrm{ps}$ each in the NVT ensemble, when data were collected for mean square displacement of the migrations. For the NVE ensemble, the number of molecules $N$, volume $V$, and energy $E$ of the system are kept constant, whereas for the NVT ensemble, the number of molecules $N$, volume $V$, and temperature of the system are kept constant. The time step of $1 \mathrm{fs}$ is taken to be constant for all the simulations of this study. The temperature was maintained by coupling the system to a temperature bath using Nose-Hoover approach [26]. From the molecular dynamics trajectories of the system we are able to estimate diffusion coefficients via the Einstein relationship.

\subsection{Diffusion coefficient}

The diffusion coefficient is estimated from the proportionality constant according to the Einstein form of diffusion based on the statistical mechanical principles [27,28]

$D=\lim _{t \rightarrow \infty} \frac{1}{6 t}\left\langle\left(r_{i}\left(t+t_{0}\right)-r_{i}\left(t_{0}\right)\right)^{2}\right\rangle$

with $D$ the diffusion coefficient; $r_{i}(t)$, the center of mass of the specie $i$ at time $t$ and $t_{0}$ all possible time origins of the production runs. 


\section{Results and discussion}

\subsection{Diffusivity inside humic acid}

The proposed humic acid model (Fig. 1) is based on the combination of the average structural unit of humic acid obtained from Sein et al. [24]. They generated a stable helical structure by building a link between the amine and carboxylate of these molecules. Our structural model of humic acid has a very similar helical feature by gradually adding several monomers to fill the lattice.

Because the positions of the toluene molecule in humic acid matrix may affect the mobility of toluene, the toluene molecule was inserted at three different positions to get the lowest energy conformation before collecting the dynamic trajectory. Considering a binary system consisting of a natural polymer, humic acid, and a penetrant, the toluene molecule, root mean square displacement from the dynamics trajectory is equal to the average of the displacement in Eq. (2) for the calculation of the diffusion coefficient.

The estimated diffusivity of toluene based on Eq. (2) in humic acid is $8.43 \times 10^{-8} \mathrm{~cm}^{2} / \mathrm{s}$ at $300 \mathrm{~K}$. This value is about an order of magnitude larger than the experimental data, the diffusivity of $6.6 \times 10^{-9} \mathrm{~cm}^{2} / \mathrm{s}$ for sorption and $1.2 \times 10^{-9} \mathrm{~cm}^{2} / \mathrm{s}$ for desorption at $298 \mathrm{~K}$ estimated by Chang et al. [5]. In general, the calculated diffusivity value becomes closer to the value obtained at the infinite simulation time as the simulation time becomes longer. However, the diffusivity did not decrease with increasing the simulation time in this case. The shorter simulation time of 10 and $20 \mathrm{ps}$ has been used in studying several chemicals in amorphous polymers [29].

To appreciate this slightly inconsistency between our calculated diffusion coefficient and the experimental estimated diffusion coefficient, we found that the density of this humic acid model is $0.92 \mathrm{~g} / \mathrm{cm}^{3}$ instead of $1.23 \mathrm{~g} / \mathrm{cm}^{3}$ as used in experimental works [5]. The density of $0.92 \mathrm{~g} / \mathrm{cm}^{3}$ was calculated from the volume of the unit cell and the mass of the atoms in the humic acid model. We propose that the lower density of the polymer will give higher diffusivity and thereby increase the mobility of penetrates.

One new humic acid model with a higher density of $1.08 \mathrm{~g} / \mathrm{cm}^{3}$ was generated from the previous humic acid model by adding one more monomer and running energy minimization calculations. We examined the effect of simulation time on the calculated values of diffusivities. Simulation time longer than $200 \mathrm{ps}$ did not change the diffusivity very much (Fig. 2). The experimental value was calculated with $200 \mathrm{ps}$ simulation.

The average diffusivity of toluene in this humic acid model is $2.93 \times 10^{-8} \mathrm{~cm}^{2} / \mathrm{s}$ at $300 \mathrm{~K}$. The diffusivity decreased with the increase of the density of humic acid matrix. The same trend was observed in three different organic diffusants in two polymers [29]. Li et al. [29] also mentioned that the difference between the calculated and experimental diffusivities could be more than four orders of magnitude in polymer systems so one may consider the calculated value reasonable if the difference is less than two orders of magnitude.

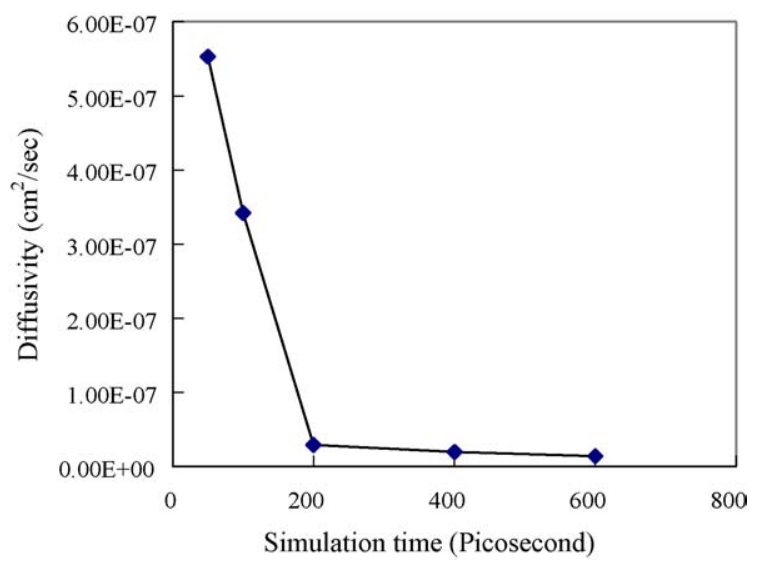

Fig. 2. Effect of simulation time on diffusion coefficient of toluene in the humic acid.

Aromatic ring of toluene molecule seems to parallel interact with the aromatic ring in the humic acid structure throughout the molecular dynamics simulation run. On analysis of each of the conformations obtained it was discovered that $\pi-\pi$ interaction occurred frequently between aromatic $\pi$ systems of humic acid matrix and benzene ring of toluene molecule.

On the basis of both molecular simulation and experimental results [5-8], we propose that the penetration of VOC is controlled by diffusion mechanism of VOC molecules in the humic substance matrix. Also the value of the diffusivity of toluene in humic substances is far less than that in the air or in the water. By using the diffusion coefficients of contaminants predicted from the molecular dynamic model and assuming the thickness of soil organic matter in the subsurface, the time needed for contaminants to penetrate soil organic matrix can be estimated.

\subsection{Diffusional activation energy}

The effect of temperature on the diffusion coefficient can be described by the Arrhenius equation $[5,7,30]$

$D=D_{0} \exp \left(-\frac{E}{R T}\right)$

where $D_{0}$ is the diffusion coefficient of the reference state and $E$, the activation energy of the diffusion process. The apparent diffusional activation energy was found by plotting $\ln D$ versus $1 / T$.

The average diffusivities of toluene in humic acid with the higher density are $1.67 \times 10^{-7} \mathrm{~cm}^{2} / \mathrm{s}$ at $350 \mathrm{~K}$ and $8.69 \times 10^{-7} \mathrm{~cm}^{2} / \mathrm{s}$ at $400 \mathrm{~K}$ shown in Table 1 and Fig. 3. Toluene sorption diffusivity increases with temperature, which is consistent with the experimental results [5]. The fact that a higher

Table 1

Calculated diffusion coefficients of toluene in the humic acid

\begin{tabular}{ll}
\hline Temperature $(\mathrm{K})$ & Diffusivities $\left(\mathrm{cm}^{2} / \mathrm{s}\right)$ \\
\hline $296 \pm 8$ & $2.93 \times 10^{-8} \pm 2.31 \times 10^{-8}$ \\
$346 \pm 10$ & $1.67 \times 10^{-7} \pm 2.37 \times 10^{-7}$ \\
$394 \pm 11$ & $8.69 \times 10^{-7} \pm 1.04 \times 10^{-6}$ \\
\hline
\end{tabular}




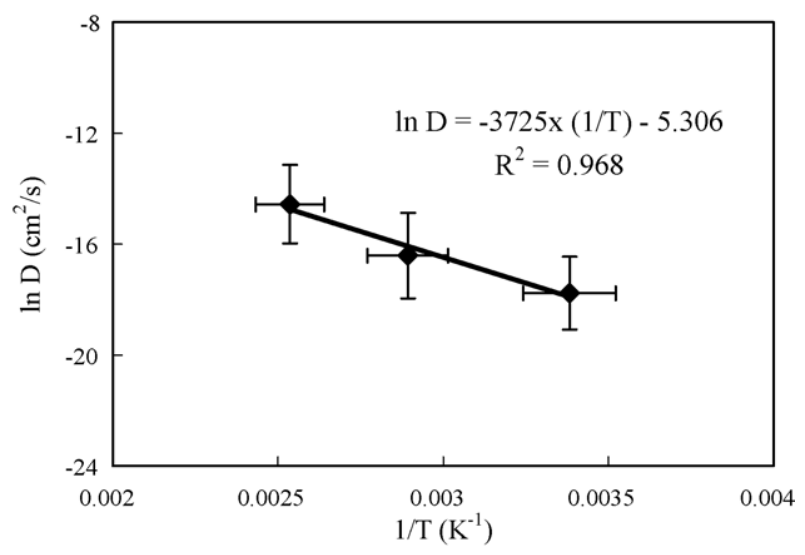

Fig. 3. $\ln D$ vs. $1 / T$ (K) for toluene sorption. Temperature ranges from 300 to $400 \mathrm{~K}$.

temperature increases a vapor's diffusion rate is attributed to the enhanced motion of both polymer segments and VOC molecules by the thermal energy. And it can be clearly observed in the process of the molecular dynamics simulation.

In Fig. 3 and Eq. (3), toluene molecules surmount the activation energy barrier of $7.4 \mathrm{kcal} / \mathrm{mole}$ when they are squeezing through the macromolecular matrix. Compared to the activation energy of toluene $10.1 \mathrm{kcal} / \mathrm{mole}$ for sorption and $15.7 \mathrm{kcal} / \mathrm{mole}$ for desorption into humic acid at $15-45^{\circ} \mathrm{C}$ estimated in [5], the value of this study is slightly lower than the experimental data. A slightly underestimated activation energy may result from the density difference discussed previously. But the similar trend between the molecular modeling and macroscopic experimental results in the finding that the molecular dynamics method may provide an alternative tool to predict molecular motion of contaminants in humic acid systems.

The computer simulation model used in our molecular dynamic simulation normally has to be refined several times and tested by different chemicals to improve its prediction capacity. Although the time for molecular dynamics calculations might be costly now, the time expense for computer simulations will be decreased with the increase of the computing power of computer systems. A more realistic structural model of the humic acid could be built by increasing the density of humic acid model and adding some water molecules in the model. The force field in the molecular dynamics simulations may be able to use the suitable interatomic force parameters for the all atoms of organic contaminants and humic acid in the near future.

\section{Conclusion}

Our work is the first study to apply molecular dynamic simulation to obtain reasonably accurate estimates of diffusion rates of the penetration of VOC molecules into a dry model humic substance. This method helps us to get useful information without the risk of applying the toxic contaminants and producing a lot of contaminants after the experiments. The trend of the diffusivities increasing with temperature is parallel to the experimental results although diffusivities are slightly overestimated.
This overestimation could be further improved by using a more realistic structural model of the humic acid and the suitable interatomic force parameters. Finally, our studies have shown that molecular dynamics simulation of volatile organic compounds in humic substances does yield meaningful results.

\section{Acknowledgments}

We gratefully acknowledge the financial support of the National Science Council of Taiwan, Republic of China (Contract NSC 91-2211-E-002-062 and NSC 93-2313-B-005-088). The authors are grateful to the National Center for High Performance Computing in Hsingchu, Taiwan for the use of a SGI supercomputer.

\section{References}

[1] C.T. Chiou, Partition and Adsorption of Organic Contaminants in Environmental Systems, John Wiley \& Sons, New York, 2002.

[2] W.P. Ball, P.V. Roberts, Environ. Sci. Technol. 25 (1991) 1237.

[3] S.M. Steinberg, J.J. Pignatello, B.L. Sawney, Environ. Sci. Technol. 21 (1987) 1201.

[4] S. Wu, P.M. Gschwend, Environ. Sci. Technol. 20 (1986) 717.

[5] M. Chang, S. Wu, C. Chen, Environ. Sci. Technol. 31 (1997) 2307.

[6] J.J. Piatt, M.L. Brusseau, Environ. Sci. Technol. 32 (1998) 1604.

[7] Y. Shih, S. Wu, J. Environ. Qual. 31 (2002) 970.

[8] Y. Shih, S. Wu, Environ. Toxicol. Chem. 21 (2002) 2067.

[9] Y. Shih, S. Wu, Environ. Toxicol. Chem. 23 (2004) 2061.

[10] Y. Shih, S. Wu, Environ. Toxicol. Chem. 24 (2005) 2827.

[11] M.L. Brusseau, P.S.C. Rao, Chemosphere 18 (1989) 1691.

[12] V. Vetter, G. Scherer, Environ. Sci. Technol. 33 (1999) 3458.

[13] F. Iino, K. Tsuchiya, T. Imagawa, B.K. Gullett, Environ. Sci. Technol. 35 (2001) 3175.

[14] J.D. Kubicki, M.J. Itoh, L.M. Schroeter, S.E. Apitz, Environ. Sci. Technol. 31 (1997) 1151.

[15] B.J. Teppen, C. Yu, D.M. Miller, L. Schäfer, J. Comput. Chem. 19 (1998) 144.

[16] S.A. Boyd, G. Sheng, B.J. Teppen, C.T. Johnson, Environ. Sci. Technol. 35 (2001) 4227.

[17] J. Farrell, J. Luo, P. Blowers, J. Curry, Environ. Sci. Technol. 36 (2002) 1524.

[18] J. Luo, J.J. Farrell, Environ. Sci. Technol. 37 (2003) 1775.

[19] S.M. Shevchenko, G.W. Bailey, L.G. Akim, J. Mol. Struct.: Theochem. 460 (1999) 179.

[20] E.A. Nantsis, W.R. Carper, J. Mol. Struct.: Theochem. 468 (1998) 51.

[21] J.D. Kubicki, S.E. Apitz, Org. Geochem. 30 (1999) 911.

[22] G.A. Davis, A. Fataftah, A. Cherkassiy, E.A. Ghabbour, A. Radwan, S.A. Jansen, S. Kolla, M.D. Paciolla, L.T. Sein Jr., W. Buermann, M. Balasubramanian, J. Budnick, B. Xing, J. Chem. Soc., Dalton Trans. (1997) 4047.

[23] S. Kolla, L.T. Sein Jr., M.D. Paciolla, S.A. Jansen, Recent Res. Adv. Phys. Chem. 2 (1998) 22.

[24] L.T. Sein Jr., J.M. Varnum, S.A. Jansen, Environ. Sci. Technol. 33 (1999) 546.

[25] Molecular Simulation Inc., San Diego, CA.

[26] W.G. Hoover, Phys. Rev. A 31 (1985) 1695.

[27] J.M. Haile, Molecular Dynamics Simulation: Elementary Methods, Wiley, New York, 1997.

[28] P. Neogi (Ed.), Diffusion in Polymers, Marcel Dekker, New York, 1996, p. 67.

[29] T. Li, D.O. Kildsig, K. Park, J. Control. Release 48 (1997) 57.

[30] J. Crank, G.S. Park, Diffusion in Polymers, Academic Press, London, UK, 1968. 